\title{
Janus particles and motors: unrivaled devices for mastering (bio) sensing
}

\author{
Beatriz Jurado-Sánchez ${ }^{1,2} \cdot$ Susana Campuzano ${ }^{3} \cdot$ José M. Pingarrón ${ }^{3} \cdot$ Alberto Escarpa $^{1,2}$ (0)
}

Received: 3 September 2021 / Accepted: 13 October 2021 / Published online: 10 November 2021

(c) The Author(s), under exclusive licence to Springer-Verlag GmbH Austria, part of Springer Nature 2021

\begin{abstract}
Janus particles are a unique type of materials combining two different functionalities in a single unit. This allows the combination of different analytical properties leading to new analytical capabilities, i.e., enhanced fluid mixing to increase sensitivity with targeting capturing abilities and unique advantages in terms of multi-functionality and versatility of modification, use, and operation both in static and dynamic modes. The aim of this conceptual review is to cover recent (over the last 5 years) advances in the use of Janus microparticles and micromotors in (bio)-sensing. First, the role of different materials and synthetic routes in the performance of Janus particles are described. In a second main section, electrochemical and optical biosensing based on Janus particles and motors are covered, including in vivo and in vitro methodologies as the next biosensing generation. Current challenges and future perspectives are provided in the conclusions section.
\end{abstract}

Keywords Janus $\cdot$ Particles $\cdot$ Micromotors $\cdot$ Biosensing $\cdot$ Electrochemical $\cdot$ Optical $\cdot$ Cellular $\cdot$ In vivo $\cdot$ In vitro

\section{Introduction}

Janus particles (JPs) are a unique type of nanomaterials that combine two or more physical or chemical properties in the same entity, resulting in unpreceded capabilities [1]. The concept "Janus Particle" to define anisotropic materials was pioneered by the Nobel Laureate Pierre-Gilles de Gennes during his lecture about "Soft matter." Among other findings, Gennes synthetized the first JP consisting of a glass bead with polar and non-polar hemispheres [2]. Later, JPs were modified with catalytic structures (such as Pt nanoparticles) for autonomous motion in solution by catalytic fuels or other external inputs [3, 4]. Since then, a myriad of JPs and Janus micromotors (JMs) composed by one or more

Beatriz Jurado-Sánchez

beatriz.jurado@uah.es

$\triangle$ Alberto Escarpa

alberto.escarpa@uah.es

1 Department of Analytical Chemistry, Physical Chemistry and Chemical Engineering, University of Alcala, Alcala de Henares E-28871, Madrid, Spain

2 Chemical Research Institute "Andrés M. del Río", University of Alcala, Alcala de Henares E-28871, Madrid, Spain

3 Department of Analytical Chemistry, Faculty of Chemistry, Universidad Complutense de Madrid, 28040 Madrid, Spain polymeric, carbon, inorganic, or organic nanomaterials have been synthetized. Although there is no clear definition of the difference between JPs and JMs, a JM can be defined as a JPs capable of moving autonomously in the solution. Rapid development in the field allows introducing multifunctional properties and capabilities for functionalization, which can be tailored by judicious selection of the type of nanomaterial used for particle or micromotor synthesis. Applications are vast in fields such as the biomedical, analytical, or environmental remediation, among others $[5,6]$. In this review, we focus on the analytical dimension of JPs and JMs for enhanced (bio)sensing. The reader is referred to current recent reviews to expand the dimension of JPs and JMs in other fields [1, 3-7].

What makes JPs and JMs unique for analytical (bio)-sensing is the asymmetric structure, which allows the integration of multifunctional features required for efficient (bio) sensing, including analyte enrichment, signal enhancement, and convenient transduction. Indeed, while asymmetry is required for efficient propulsion of JMs and enhanced fluid mixing, in the case of JPs, it allows targeted immobilization of receptors such as aptamers and antibodies for an improvement in overall analytical properties, including selectivity. This ultimately results in the overall improvement of analytical sensing features, which is of paramount significance in biomedical diagnosis or food control [3]. Figure 1 shows 
a)
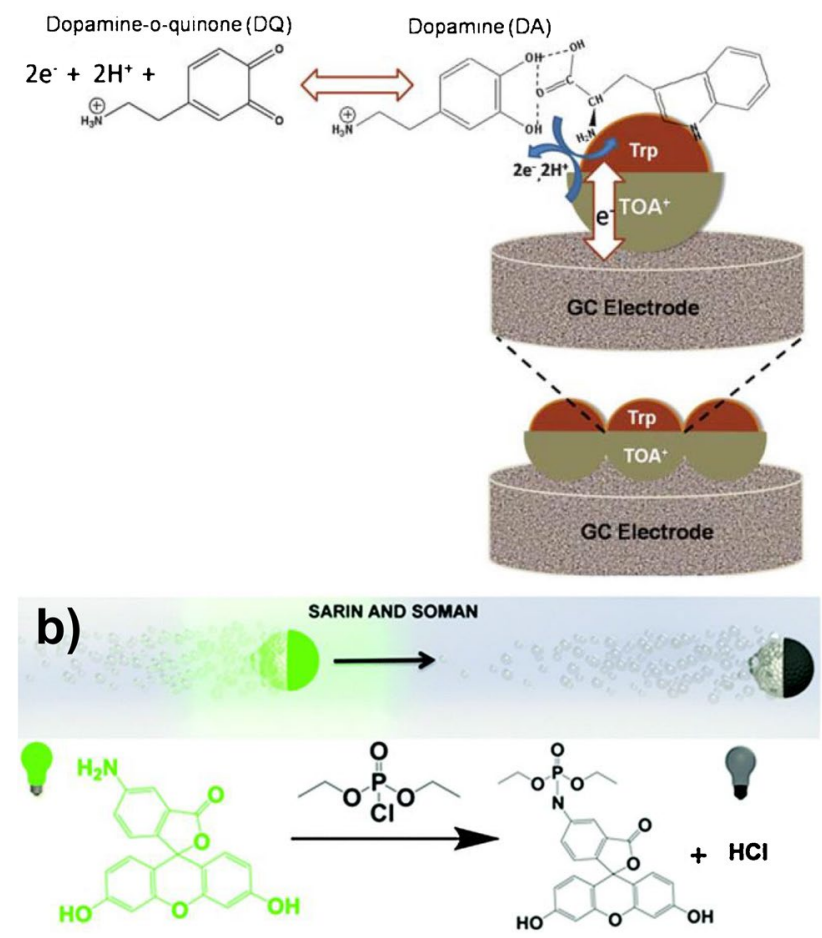

Fig. 1 JPs and JMs enhanced (bio)-sensing. a GCE modified with $\mathrm{TOA}^{+} /$tryptophan Janus gold nanoclusters for the electrocatalytic oxidation of DA. b $\mathrm{SiO}_{2}$-FL/Pt micromotors for OFF-ON fluorescence detection of chemical warfare agents Reprinted with permission from ref. [8] (a) and ref. [9] (b), respectively

two representative examples of JPs and JMs with multifunctional capabilities for improved biosensing. Biji and Patnaik [8] exploited the use of Janus gold nanoclusters, modified through non-covalent interactions with the ligands tetraoctylammonium cation $\left(\mathrm{TOA}^{+}\right)$and tryptophan, as modifiers in a glassy carbon electrode (GCE) for dopamine (DA) sensing (Fig. 1a). In this method, the presence of tryptophan at the peripheral Janus cluster hemisphere attracted a larger amount of DA towards the nanocluster surface through $\mathrm{H}$-bonding interactions thus allowing the use of the electrode as a pre-concentrating layer and enhancing the electrocatalytic oxidation of DA (see blue arrows). The resulting sensor exhibited antifouling properties and detected DA in the sub-nanomolar concentration range (limit of detection, LOD of $0.5 \mathrm{nM}$ ) in contrast with $6400 \mathrm{nM}$ provided by the bare GCE using differential pulse voltammetry (DPV). Unfortunately, no comparative experiments were performed by nanostructuring the GCE, e.g., with AuNPs. Figure 1b displays silica $\left(\mathrm{SiO}_{2}\right) / \mathrm{Pt} \mathrm{JMs}$ loaded with fluorescein amine (FLA) which were used for the OFF-ON detection of the chemical warfare agent simulant diethyl chlorophosphate (DCP) [9]. The Janus structure allowed the encapsulation of the fluorescence FLA, which was quenched in the presence of DCP in a concentration dependent manner. The catalytic Pt layer promoted the efficient micromotor motion and fluid mixing thus decreasing the detection times. Indeed, no fluorescence quenching was observed after $1 \mathrm{~min}$ in solutions contaminated with DCP using static (not moving) micromotors, while only $10 \mathrm{~s}$ was needed in moving mode. In both examples, the asymmetric structure of JPs allowed the integration of multifunctional capabilities benefiting optical and electrochemical biosensing.

In view of the potential of Janus structures in analytical (bio)-sensing, the aim of this review is to cover recent advances (last 5 years) on the use of JPs and JMs to enhance analytical (bio)-sensing. The first more fundamental section reports the role of different synthetic routes in the multifunctional properties of the resulting nanoentities, with special emphasis in biocompatibility and operational ability in complex (bio)media. The second more applied section covers electrochemical and optical biosensing using JPs and JMs including in vivo and in vitro methods as the next biosensing generation. Current challenges and future perspectives are discussed in the final section.

\section{JPs and JMs: fabrication routes and attributes pursued}

\section{Synthesis}

Many materials have been explored for synthesis of JPs and JMs. Such materials and their designs play a crucial role in the analytical (bio)-sensing performance, affecting both functionalization and biocompatibility. We focus here only on the most applied nanomaterials and synthetic routes. Table 1 summarizes the synthesis strategies and related (bio)-analytical applications. Polymeric, silica, and inorganic nanomaterials (including reactive particles) and their combinations are the most employed, prepared either by controlled self-assembly, masking, or phase separation (see schematic of these synthetic routes in Fig. 2).

Self-assembly rely on the autonomous assembly of blockcopolymers into similar structures. The size and shape of the resulting Janus structures can be tailored by tuning the interactions among individual blocks, the fraction, and the interactions with the surrounding media. The synthesis can be made in bulk or in solution. For example, amphiphilic polymeric JPs have been prepared by assembly in solution of poly(ethylene oxide)-b-poly(4-vinylpyridine) functionalized yttrium hydroxide nanotubes with hydrophobic divinyl cross-linker and a hydrophobic free-radical initiator [10]. 2D nanomaterials wrapped JMs have been prepared by selfassembly of sulfhydryl-modified graphene with gold sputtered silica nanoparticles, followed by controlled growth/ assembly of platinum, iron oxide nanoparticles (NPs), and 
Table 1 Janus particles and micromotors: materials, synthesis, and applications

\begin{tabular}{llll}
\hline Material & Examples & Synthesis & Application \\
\hline Polymers & $\begin{array}{l}\text { Polycaprolactone } \\
\text { Polystyrene }\end{array}$ & $\begin{array}{l}\text { Self-assembly } \\
\text { Masking } \\
\text { Phase separation }\end{array}$ & $\begin{array}{l}\text { Electrochemical and optical biosensing } \\
\text { (in vivo/in vitro) }\end{array}$ \\
Silica & Mesoporous silica & $\begin{array}{l}\text { Self-assembly } \\
\text { Masking } \\
\text { Self-assembly }\end{array}$ & Electrochemical biosensing (in vitro) \\
Masking & Electrochemical biosensing (in vitro) \\
Inorganic & Gold, silver & Mgsking & $\begin{array}{l}\text { Electrochemical and optical biosensing } \\
\text { (in vivo/in vitro) } \\
\text { Cellular approaches }\end{array}$ \\
\hline
\end{tabular}

Fig. 2 Synthetic routes of JPs and JMs. Reprinted with permission from ref. [7]

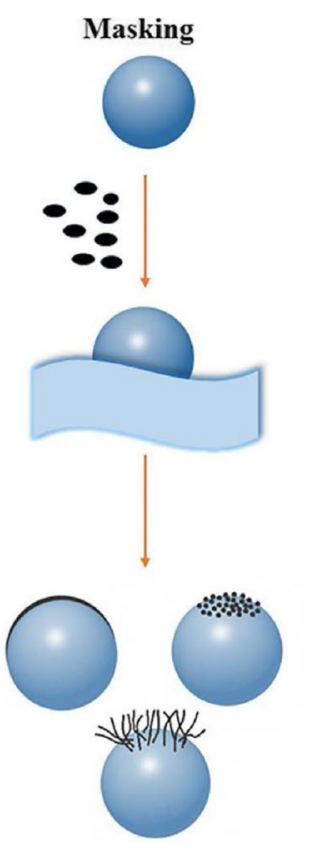

quantum dots (QDs) [11]. The main advantage of selfassembly for the preparation of JPs and JMs is the precise control of the morphology, shape, and structure of the resulting structures. Yet, the technique is mainly restricted to polymeric materials and the cost makes it not feasible for large scale-up processes [12].

Masking is a term used to define many routes for asymmetric decoration/deposition of a material in NPs used as template. Usually, JPs are either bounded to a solid substrate, which is removed after the modification, or trapped in the interface between two fluid phases [13]. The use of solid substrates relies in the formation of a monolayer of particles (polystyrene, $\mathrm{Mg}$, etc.) on a glass slide followed by the deposition of a half-layer or gold, platinum, etc. by chemical/physical vapor deposition, atomic layer deposition, etc. The glass slide can be set as an angle, i.e., glancing angle deposition, to facilitate the formation of the asymmetric layer. This technique has been used for the preparation of Au-coated polystyrene JPs [14] or Mg/Au JMs used
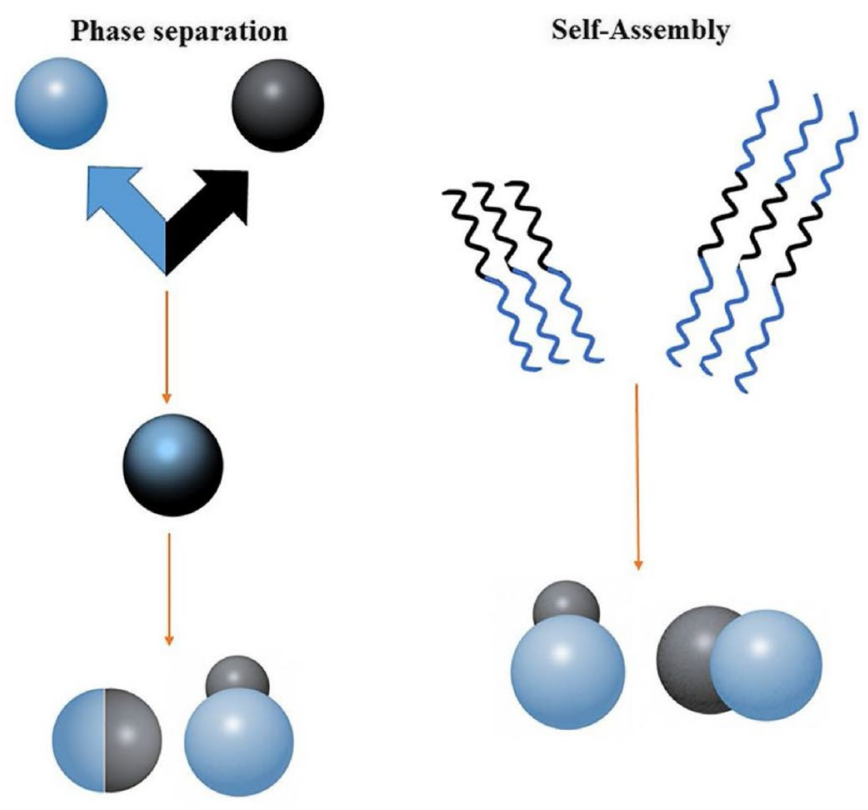

in electrochemical sensing [15]. With this approach, the amount of JMs produced is limited by the particles dispersed in the solid substrates thus preventing large-scale synthesis. Masking in solution is denoted as pickering emulsion and allows the large-scale JP synthesis. In this case, NPs or polymers are trapped between the liquid-liquid interface of immiscible fluids, followed by chemical modification in the aqueous phase [16, 17]. Granick was one of the first pioneer in the use of this methodology, reporting the fabrication of JPs by dispersing silica particles in paraffin wax and mixing the resulting solution with water. This promoted the trapping and locking of the silica NPs in the oil-water interface which was followed by chemical modification to obtain cationic or anionic half-modified JPs [18].

Phase separation is the most used synthetic method for the preparation of JPs and JMs, either in solution or using microfluidic devices. Yet, it is mostly applied for polymeric materials but allows the introduction of metallic NPs or other components [19-21]. For example, polystyrene/poly(methyl 
methacrylate) JPs have been prepared by dispersion of such polymers in toluene and mixing with an aqueous solution containing polyoxyethylene nonylphenyl and a surfactant. The slow evaporation of the solvent resulted in the generation of JPs with different hemispheres formed by each individual polymer in a single unit [22]. Magneto-catalytic JMs have been prepared by mixing an organic phase containing polycaprolactone (PCL), platinum NPs, and iron oxide NPs with an aqueous phase containing a surfactant and GQDs. After mixing, an oil-in-water emulsion was generated, in which interface PCL drops trapping the NPs and the QDs were formed. After solvent evaporation, the PCL drops reduced its size, while trapping the QDs all over the particle and the NPs in one size of the structure due to electrostatic repulsion with the polymers. The resulting micromotors were used for optical sensing and can operate by the action of chemical fuel or magnetic fields [23].

Bipolar electrochemistry is an alternative concept to break the symmetry of particles in solution, generating an asymmetric patch. In other words, polarized base particles are placed in a special set-up or chamber containing two connected electrodes for the application of strong electric field. In this way, tailored electrochemical reactions can carry out on the opposite (half-side) of the particles, resulting in the generation of JPs and JMs [24].

To summarize, the synthetic route plays a critical role in further (bio)sensing applications, affecting both performance and biocompatibility. Self-assembly and solid substratesbased approaches result in low-yield syntheses (or relatively low number of NPs) but allow tailoring the composition. Pickering emulsions and phase separation, on the contrary, can be used for large-scale synthesis to translate basic science into industrial and point-of-care applications.

\section{Operation in real (bio)-media: propulsion and biocompatibility}

Analytical methods in biological domains require full biocompatibility of the JPs and JMs in this latter case including composition and propulsion mode. Of course, biocompatibility is of crucial importance in in vivo biosensing where the Janus NPs should be administered to the human body. First, biocompatible materials should be ideally employed. Another key point to consider is the biodegradability of the materials into non-harmful products, which is of paramount importance in in vivo bioimaging and cellular sensing, and is compatible with the principles of green chemistry [25]. In the case of JMs, biocompatible propulsion schemes are needed, as the oxygen peroxide or other fuels used traditionally are toxic to human cells avoiding its direct application in whole blood. With this aim, alginate [26, 27], chitosan [28], cellulose, mesoporous silica, and biodegradable polymers such as PCL [29] have been used for the preparation of JPs and JMs. A group of biocompatible JMs is composed by reactive $\mathrm{Mg}$ and $\mathrm{Zn}$ particles that can react with water and body fluids generating hydrogen bubbles for autonomous propulsion while the body of the micromotor dissolves. In addition, the released products are non-toxic, holding considerable promise for cellular and in vivo methods [30, 31]. Another potential approach for these biocompatible JMs is their use to aid electrochemical detection, replacing the classical fuel peroxide, which can greatly interfere with electrochemical detection. A second group compromise light and magnetic-propelled JMs, which can propel in body fluids (including whole blood) in optical detection schemes. For example, a PCL micromotor encapsulating magnetic nanoparticles and biocompatible QDs can move in serum and blood for the detection of heavy metals and toxins. Biocompatibility assays revealed the full compatibility with the fluids, and the micromotor can be degraded using natural lipases [32].

It should be also considered that once in contact with body fluids, blood protein adhesion and other components from the immune system will interact with JPs and JMs, preventing further in vivo operation and leading to the so-called biofouling effects. This can hamper analytical performance and avoid adequate in vivo operation [33]. To avoid this, JPs and JMs can be functionalized with external layers such as protein corona, natural proteins [34], or hydrogels [35], among others.

\section{Sensing and biosensing with JPs and JMs}

\section{Electrochemical (bio)sensing}

JPs and JMs have been exploited in electrochemical sensing and biosensing [6, 36-38]. While JPs can increase the loading of biomolecules on the electrode surface thus amplifying the electrochemical response [39], the self-propulsion of the JMs improves, besides the sensitivity, the kinetics of the assay. Moreover, the products generated during their movement can be exploited to degrade and sense non-electroactive toxic compounds (pesticides or emerging contaminants) into detectable electroactive by-products. However, to date JPs have been exploited more than Janus engines in (bio)-sensing probably due to the interference caused by fuels $\left(\mathrm{H}_{2} \mathrm{O}_{2}\right)$ or radicals generated by the most used catalytic JMs. Nevertheless, the discovery of redox JMs that use the body of the motor as a fuel itself for self-propelling in aqueous media has opened new avenues in this area [36, 40].

Villalonga's group reported the use of $\mathrm{Au}$-mesoporous silica Janus nanoparticles (Au MS JNPs) and bifunctionalized JPs for catalytic [41] and affinity [42, 43] electrochemical biosensing. The first work reported Au MS JNPs dually functionalized with covalently immobilized horseradish 
peroxidase (HRP) as enzymatic signaling element on the mesoporous silica face, and with streptavidin (Stv) and polyethylenglycol chains as biorecognition and solubilizing agents, respectively, on the Au face (Au-MS JNPs-HRP-StvPEG) (Fig. 3a). Such functionalized JPs, with biorecognition-signaling ability, were successfully employed to recognize biotin on gold surfaces in a proof-of-concept application by monitoring the HRP reduction of $\mathrm{H}_{2} \mathrm{O}_{2}$ using cyclic voltammetry (CV). Two years later, the same group reported the use of Au MS JNPs with glucose oxidase (GOx) and HRP immobilized on the Au and mesoporous silica faces, respectively, in connection with carbon nanotubes (CNTs) [41] as GCE modifiers to construct an amperometric glucose biosensor (Fig. 3b). The resulting biosensor achieved a LOD of $360 \mathrm{nM}$ and was employed for the analysis of glucose in commercial soft drinks.

More recently, the same group used bifunctionalized JPs as biorecognition-signaling elements in an amperometric signal-on aptasensor for the determination of carcinoembryonic antigen (CEA) [43]. In this strategy, the JNPs were functionalized with HRP (signaling element) on the silica surface, and with a dually biotin thiol-specific hairpin structure aptamer (biorecognition element) on the Au face (Fig. 4). The aptamer bound to the bifunctionalized JNPs was unfolded in the presence of CEA releasing the biotin residues so that the CEA-JNP complex could be captured by avidin-modified $\mathrm{Fe}_{3} \mathrm{O}_{4} @ \mathrm{SiO}_{2}$ NanoCaptors ${ }^{\circledR}$ (Fig. 4). The amperometric detection carried out in the presence of $\mathrm{H}_{2} \mathrm{O}_{2}$ /hydroquinone (HQ) upon deposition of the magnetic nanoconjugates on the working electrode surface of a screen-printed carbon electrode (SPCE) allowed detection of $1.2 \mathrm{pM}$ of CEA standard. The method was applied to perform the determination in a spiked commercial serum sample.

He and Shuang's group used JPs as GCE modifiers to develop label-free electrochemical aptasensors [39, 44]. The affinity reactions were monitored by measuring the decrease in the DPV reduction peak current measured in the presence of $\left[\mathrm{Fe}(\mathrm{CN})_{6}\right]^{4-/ 3-}$ due to the hindered diffusion of the redox probe to the electrode. One of the

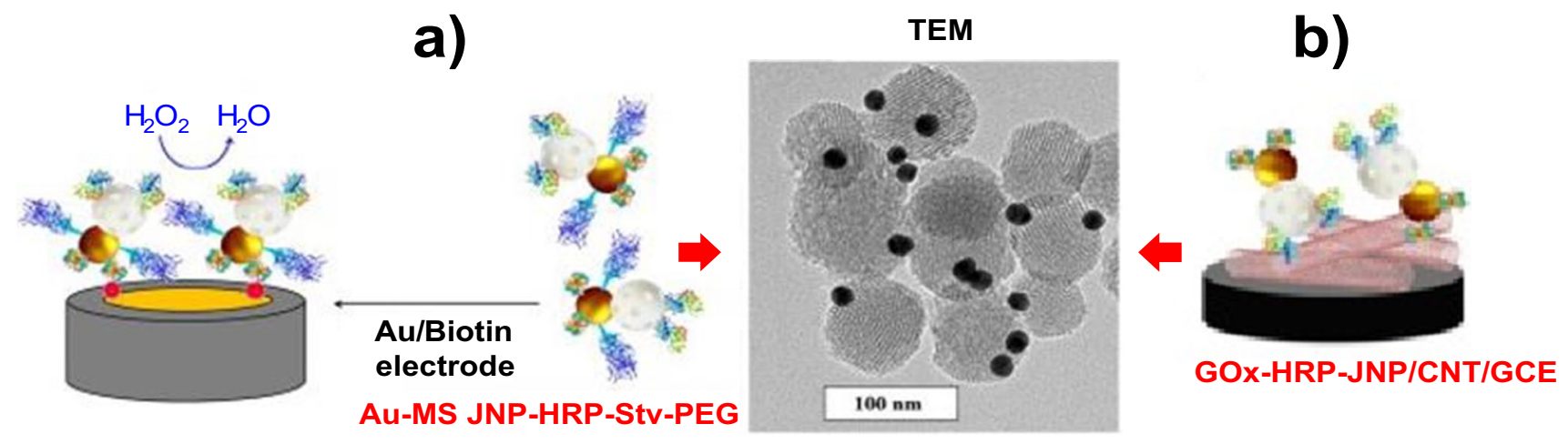

Fig. 3 Au MS JNPs dually functionalized with HRP and Stv and polyethylenglycol chains used as biorecognition-signaling system (a) or with GOx and HRP enzymes as electrode modifiers (b) for affinity and catalytic electrochemical biosensing, respectively. A representa- tive transmission-electron microscopy (TEM) image of these Au MS JNPs is also shown. Reprinted with permission from ref. [42] (a) and ref. [41] (b)

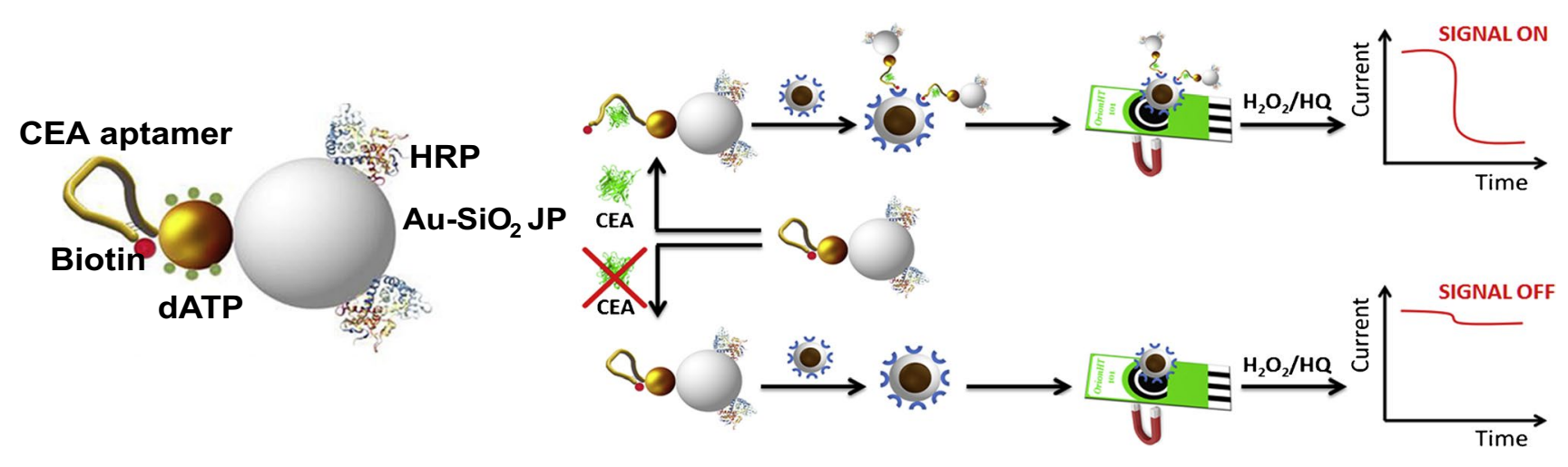

Fig. 4 Use of bifunctionalized JNPs as biorecognition-signaling elements in an amperometric signal-on CEA aptasensor. Reprinted with permission and adapted from ref. [43] 
Fig. 5 Aptasensor for the determination of OTA constructed by coupling aptamer/ $\mathrm{NH}_{2} \mathrm{JPs}$ with COOH-GN/GCE. Reprinted with permission and adapted from ref. [44]

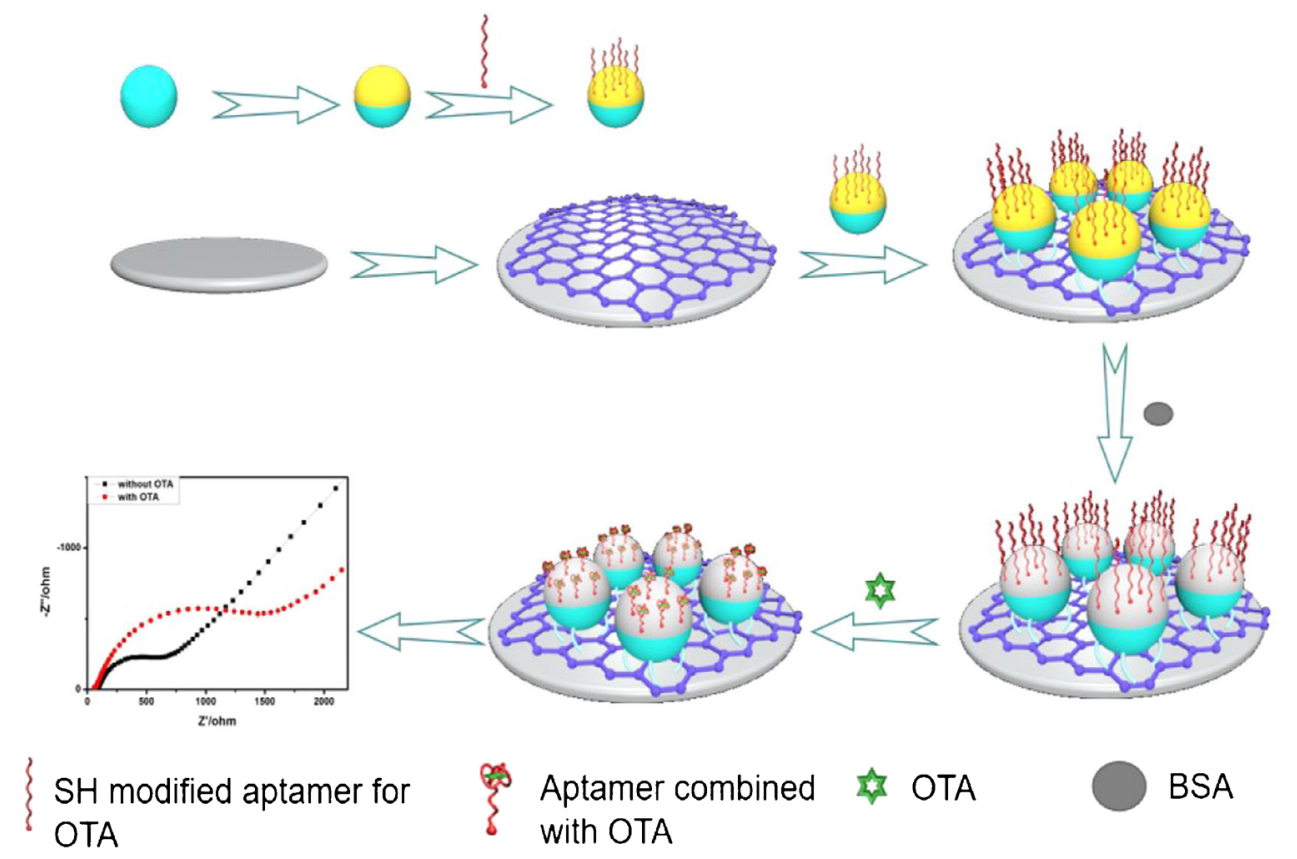

works reported an aptasensor for the determination of ractopamine (RAC) and involved the use of JPs with one hydrophobic side (octadecanethiol) to connect to the GCE surface, and one hydrophilic side to immobilize $\mathrm{Au}$ NPs, followed by the attachment of the thiolated specific aptamer [39]. A more recent method used aptamer $/ \mathrm{NH}_{2}$ JPs for the electrochemical determination of Ochratoxin A (OTA) [44]. In this approach, gold was coated on the hemispherical surface of the aminated polystyrene particles to immobilize the thiolated OTA aptamer and the exposed amino groups of the other hemisphere allowed the covalent immobilization of the JPs through carbodiimide/ succinimide (EDC/NHS) chemistry onto a GCE modified with carboxylated graphene (COOH-GN) (Fig. 5). Both aptasensors achieved similar LOD values $\left(3.3 \times 10^{-14}\right.$ and $1 \times 10^{-14} \mathrm{M}$ for RAC and OTA, respectively) which were competitive in comparison with other reported methods. The aptasensors were employed to analyze spiked human urine (RAC) and wine (OTA) samples.

The efficient self-propulsion of certain JPs allows them to mix with liquids much better than static particles making it possible to perform dynamic electrochemical sensing with improved efficiency in the absence of external stirrers [45]. For instance, $\mathrm{Au}$ and $\mathrm{Mg} \mathrm{JPs}$ are propelled in aqueous $\mathrm{NaCl}$ medium by corrosion reactions that generate bubbles on one side and propel JPs in the opposite direction. By combination of galvanic and pitting corrosion processes, the gold layer coating along with the presence of chloride ions in the medium induces the dissolution of the $\mathrm{Mg}(\mathrm{OH})_{2}$ passivation layer in the $\mathrm{Mg}$ particle and subsequently the spontaneous redox oxidation reaction between $\mathrm{Mg}$ and water to generate directional hydrogen bubble propulsion (for enhanced fluid mixing) and $\mathrm{OH}^{-}$ions for alkaline degradation (Fig. 6a) [36].

Thus, these JMs exert a dual function as "autonomous stirrers" imparting effective micromixing and enhanced mass transfer convective transport using microliter volumes and as "natural enzyme mimics," inducing localized pH gradients that can be exploited in the degradation, by alkaline hydrolysis, of non-electroactive analytes. The enhanced fluid mixing, measured by calculating the mean square displacement of bead microtracers, evidenced a much higher displacement induced by moving micromotors as compared to Brownian motion. In fact, the $\mathrm{pH}$ value can be controlled with the amount of micromotors added to the solution. The $\mathrm{pH}$ value increased from 9 to 12 with the micromotor suspension volume from 1 to $4 \mu \mathrm{L}$, respectively. The use of larger volumes of micromotor suspension provoked poorer $\mathrm{S} / \mathrm{N}$ ratios [36]. These interesting characteristics make them ideal to perform electrochemical measurements on microvolume samples at screen-printed electrodes [36, 40].

To date, JMs have been used much more in optical sensing than in electrochemical biosensing. Cinti et al. [15] and Rojas et al. [46] exploited the use of bubble generating Janus microengines with screen-printed electrodes using aqueous $\mathrm{NaCl}$ (which also acted as supporting electrolyte) media. In these strategies, the movement of micromotors generated a greatly enhanced fluid transport, which led to a significant improvement in sensitivity ( $15-20$-fold) compared to the static approach, and to a rapid detection ( $\sim 5 \mathrm{~min})$ and degradation (assisted by hydrogen microbubbles and hydroxyl ions generated during the micromotors self-movement) of toxic non-detectable species to easily detectable electroactive non-hazardous compounds (Fig. 6a). In these methods, 

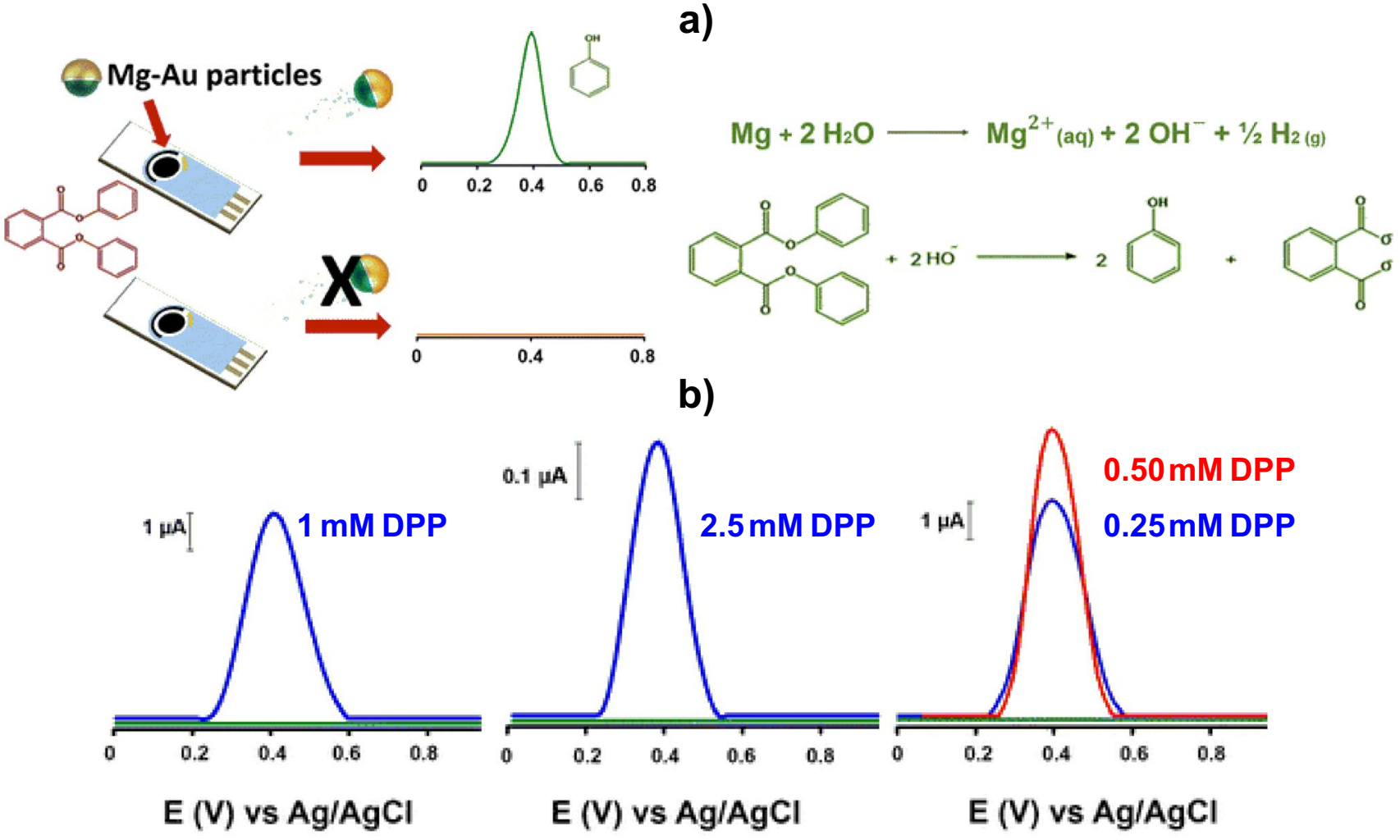

Fig. 6 a $\mathrm{Mg} / \mathrm{Au} \mathrm{JMs}$-based electrochemical strategy for the simultaneous degradation/detection of DPP and reactions involved in the degradation. b DPV voltammograms obtained from left to right for

Au-Ni-Mg (magnetically confined on the surface of SPEs through the $\mathrm{Ni}$ interlayer) or unconfined $\mathrm{Mg}-\mathrm{Au} \mathrm{JMs}$ were used for the determination of the organophosphorous (OP) nerve agent paraoxon, or of diphenyl phthalate (DPP) using DPV in spiked raw food (milk, whiskey) and biological (human plasma) samples (Fig. 6b). Although these attractive strategies can be exploited for the detection of traces of other environmental pollutants or for alkaline-assisted electrocatalytic oxidation reactions, they are limited by the relatively short lifetime of the micromotors as they are dissolved after $5 \mathrm{~min}$ of movement in the sample. Pumera employed $\mathrm{Mg} / \mathrm{Pt}$ micromotors together with SPCE for enhanced glucose biosensing, with the enhanced mixing induced by the micromotors increasing the overall electrochemical signal [47]. Bipolar electrochemistry has also been applied for the synthesis of Mg-based micromotors with dynamically controlled motion and analytical operations. Such JMs can simultaneously move while emitting light due to the strong redox reactivity of $\mathrm{Mg}$, as illustrated with the model chemiluminescence mediator $\mathrm{Ru}(\mathrm{bpy})_{3}{ }^{2+}$, which can be exploited for (bio)-sensing applications [48].

It is worth to note also that Moo and Pumera [49] demonstrated the potential of particle-electrode impact voltammetry for the real-time tracking and surveying of JM motion milk, whiskey, and human plasma samples unspiked (green) and spiked (blue and red) with DPP at the indicated concentrations. Reprinted with permission and adapted from [46]

in solution even in a microfluidic channel [50], which may be relevant to address future applications in electrochemical sensing and biosensing.

\section{Optical biosensing and bioimaging}

\section{In vitro biosensing approaches}

JPs and JMs offer considerable promise for optical biosensing where the spatial separation of probes or other components can help to avoid interferences among different sensing units, which is of relevance in protein-rich media. In addition, the self-propulsion of JMs can add a new dimension in optical biosensing associated with increased analytical performance by the enhancement in the fluid mixing.

Gold-coated polystyrene JPs have been used in surfaceenhanced Raman scattering (SERS) for cancer cell sensing. Polystyrene particles were coated with a thin gold layer, followed by the generation of a rough coral-like Au layer by oxygen plasma etching. The dual nature of the JPs allowed functionalization of the polystyrene hemisphere with antiHER-2 antibodies for attachment to breast cancer cells, while the rough Au hemisphere provided SERS hotspots for detection [51]. In a similar concept, commercial fluorescence 
a)

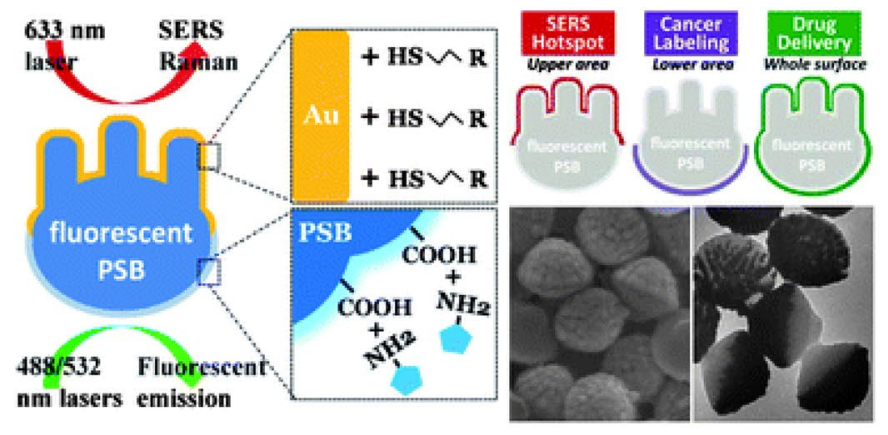

b)

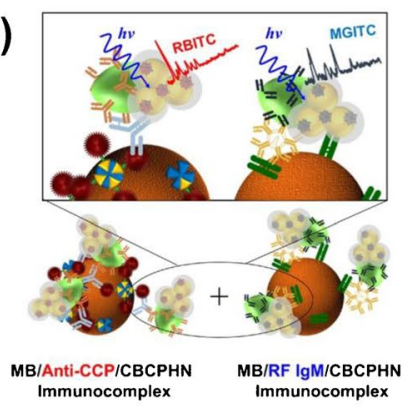

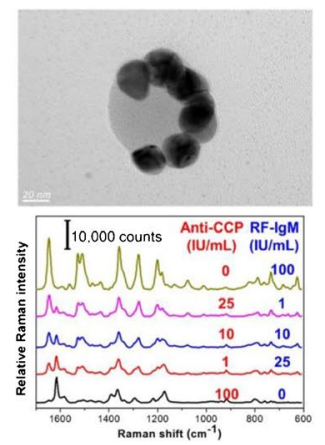

Fig. 7 SERS sensing using JPs. a Anti-CCD 4 modified fluorescence polystyrene beads (PSB) half-modified with gold for enhanced SERS sensing of cancer cells, concept, and TEM images of the particles and (b) compartmentalized bimetal cluster-poly(aniline) hybrid nanostructures (CBCPHNs) as tags for SERS-sensing of RA, schematic of

labelled polystyrene beads were modified with carboxylic groups and a half-gold layer for cancer cell targeting, SERS sensing, and imaging (Fig. 7a). The free polystyrene hemisphere was modified with anti-CD44 to target overexpressed CD44 glycoproteins in cancer cells. The JPs exhibited 12-fold cancer targeting ability and 10-fold enhancement in SERS signal compared with smooth gold nanoparticles [52]. Au-Ag-polyaniline (PA) JPs have been used as SERS tags for diagnosis of rheumatoid arthritis (RA) (Fig. 7b). The bimetallic JP nanoclusters were prepared by dye-induced aggregation of AuNPs followed by AgNPs. Next, surfacetemplated polymerization was used to decorate one hemisphere with PANI. The PANI hemisphere was modified with anti-CCP antibodies and rheumatoid factor immunoglobulin M. For the multiplexed assay, MB were modified with the same antibodies for the capture of RA autoantibodies, followed by tagging with the Au-Ag-PA Janus nanohybrids for subsequent SERS detection exploiting the Au and Ag NPs as hot-spots. Successful multiplexed detection of anti-CCP and rheumatoid factor immunoglobulin $\mathrm{M}$ was achieved with LOD of 0.7 and $0.9 \mathrm{IU} / \mathrm{mL}$, respectively [53].

Silica nanoparticles half-coated with a hematite layer $\left(\gamma-\mathrm{Fe}_{2} \mathrm{O}_{3}\right)$ display enzyme-like activity as illustrated in the colorimetric detection of glucose and $\mathrm{H}_{2} \mathrm{O}_{2}$. Figure 8 a shows as GOx-modified JPs can detect glucose in serum by using the colorimetric substrate 3,3',5,5'-tetramethylbenzidine (TMB). The reaction mechanism relied on $\mathrm{H}_{2} \mathrm{O}_{2}$ interaction with the JP surface, followed by decomposition and $\mathrm{OH}$ radicals' generation and TMB oxidation into a blue colored product in a concentration dependance manner. The linear range obtained with this method for glucose spanned from 10 to $20 \mu \mathrm{M}$, with a LOD of $3.2 \mu \mathrm{M}$ [54]. Such proof-ofconcept applications demonstrate the applicability of multifunctional Janus structures for tailored analytical (bio)sensing. JP emulsions represent a novel way to perform biosensing as illustrated by Swager et al. for the detection the multiplexed assay, SEM image, and corresponding SERS signals. RBITC rhodamine B isothiocyanate, MGITC malachite green isothiocyanate. Reprinted and adapted with permission from ref. [52] (a) and ref. [53] (b)

of Listeria monocytogenes. A block copolymer, poly-TCO, with a hydrophobic polystyrene block and a hydrophilic polyacrylic acid block was used to prepare the Janus droplet mixture, which also contained Listeria antibodies. The principle of detection was based in the aggregation of the droplets after binding of the droplet-immobilized antibodies to Listeria. To perform detection, specific fluorescence dyes were added in solution and the subsequent changes of emission in the presence of Listeria were monitored [55]. Similarly, Janus emulsions have been used for biosensing of anti-SARS-CoV-2 spike antibodies (Fig. 8b) [56]. Janus emulsions were prepared by mixing immiscible hydrocarbon and fluorocarbon oils with water. The resulting water/ organic phase interface was functionalized with a secondary antibody of IgG protein and SARS-CoV-2 spike receptor binding domain, resulting in two Janus emulsions. In the presence of the anti-SARS-CoV-2 spike IgG antibody, the droplets agglutinated. The extent of agglutination was monitored by taking optical images and quantitative fluorescence spectra in the presence of Lumogen F Orange 240 dye. The method achieved a LOD of $0.2 \mu \mathrm{g} / \mathrm{mL}$.

JM-based optical biosensing involves mainly fluorescence detection. The dual Janus nature allows simultaneous detection (via encapsulation or modification of the micromotor with the detection probes) and enhanced mixing to decrease the overall response time while improving analytical performance. Two broad strategies have been adopted, with most designs based on catalytic propulsion using peroxide fuel. Recent works exploit fuel-free schemes such as magnetic propulsion to improve biocompatibility in real samples while facilitating in vivo applications. Magneto-catalytic Janus micromotors loaded with phenylboronic acid-modified graphene QDs have been used for ON-OFF detection of Escherichia coli endotoxin in sepsis diagnosis applications. The JMs were prepared using an oil-in-water emulsion for the encapsulation of the modified QDs, Pt, and iron oxide 
a)
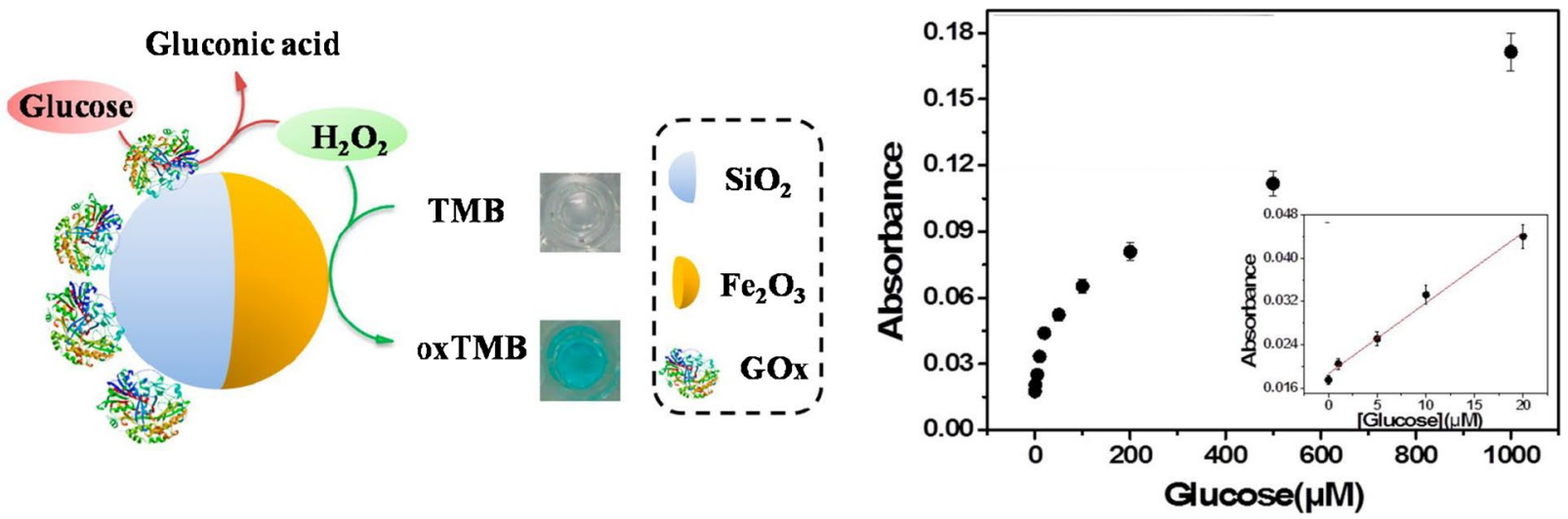

b)

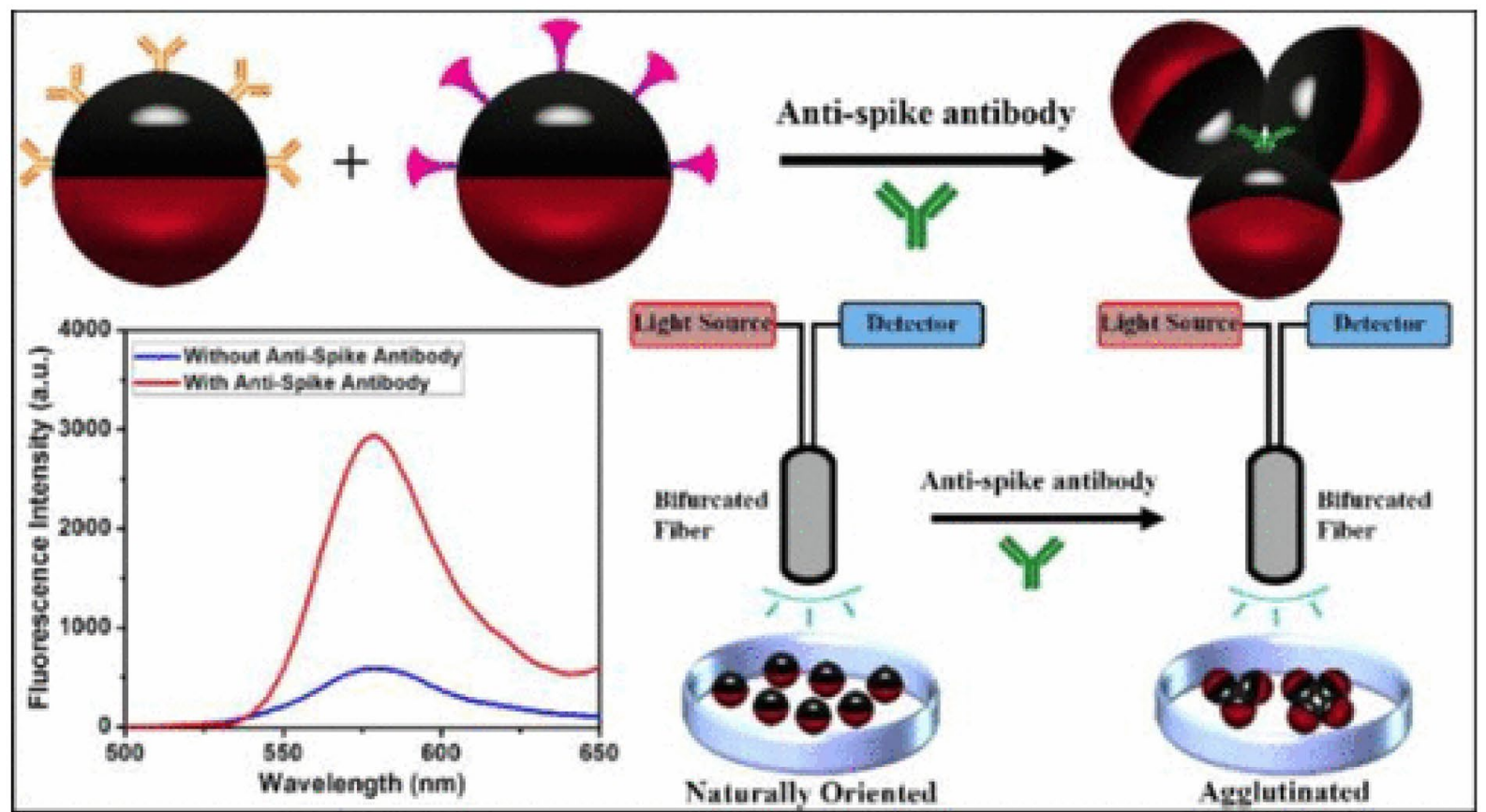

Fig. 8 Colorimetric and fluorescence biosensing using JPs. a GOx functionalized silica-hematite JPs for colorimetric detection of glucose; schematic of the detection, absorbance spectra in the presence on increasing concentrations of glucose and corresponding calibra- tion plot. b Janus emulsion biosensing of anti-SARS-CoV-2 spike antibody by agglutination assay. Reprinted and adapted with permission from ref. [54] (a) and ref. [56] (b)

JMs sensing strategies lack selectivity due to all endotoxins contain a KDO residue. To solve the problem, the micromotor structure was redefined by using PCL JMs loaded with $\mathrm{WS}_{2}$ for subsequent attachment of a rhodamine labelled affinity peptide specific for Escherichia coli endotoxin. The OFF-ON strategy is displayed in Fig. 9a. The $\mathrm{WS}_{2}$-loaded micromotors were incubated with a solution containing the fluorescence labelled affinity peptide which entered the micromotor and interacted with the $\mathrm{WS}_{2}$, this resulting in the fluorescence quenching of the solution. In the presence of the Escherichia coli endotoxin, the high affinity of the peptide probe provoked the release from the micromotor into the solution, resulting in an increase of the fluorescence in the solution in a concentration dependent manner. The method treatment [57]. Despite their efficiency, the above mentioned 
a)

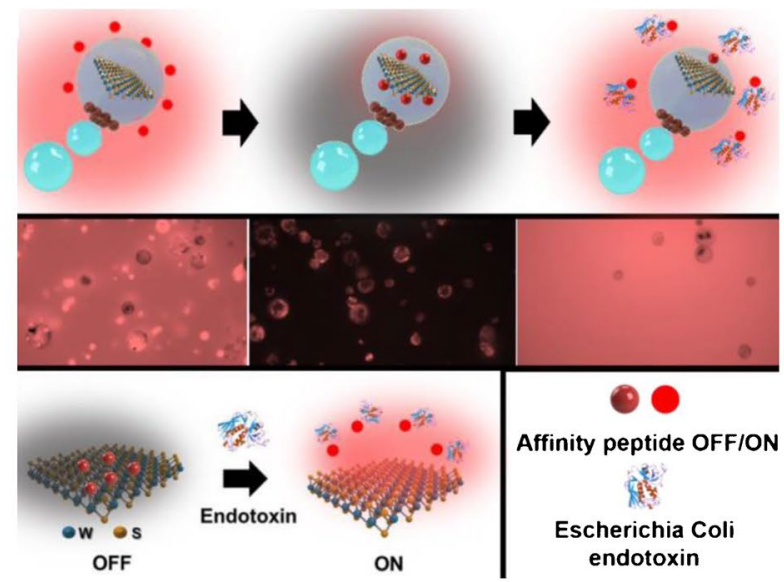

Fig. 9 Catalytic JMs for optical sensing. a $\mathrm{WS}_{2} / \mathrm{PCL} / \mathrm{Pt}$ Janus micromotors functionalized with a specific affinity peptide for OFF-ON Escherichia coli detection. Top part shows the schematic of the structure from peptide loading (left) and release in the presence of the spe-

was highly selective, with no fluorescence increase in the presence of Salmonella Enterica endotoxin. The strategy was validated against the gold standard Limulus Amebocyte lysate in a specific culture, with excellent match. A LOD of $120 \mathrm{pM}$ was obtained, which allows detection in real clinical settings [58]. PCL micromotors prepared according to a similar structure were loaded with a covalent organic framework for ON-OFF fluorescence detection of nitroaromatic explosives in water [59].

Catalytic JMs prepared by self-assembly are also attractive for biosensing applications related with clinical diagnosis. 2D nanomaterials (graphene, graphdiyne, and black phosphorus) wrapped silica-gold micromotors have been combined with a fluorescence labelled affinity peptide specific to cholera B toxin subunit for the detection of such

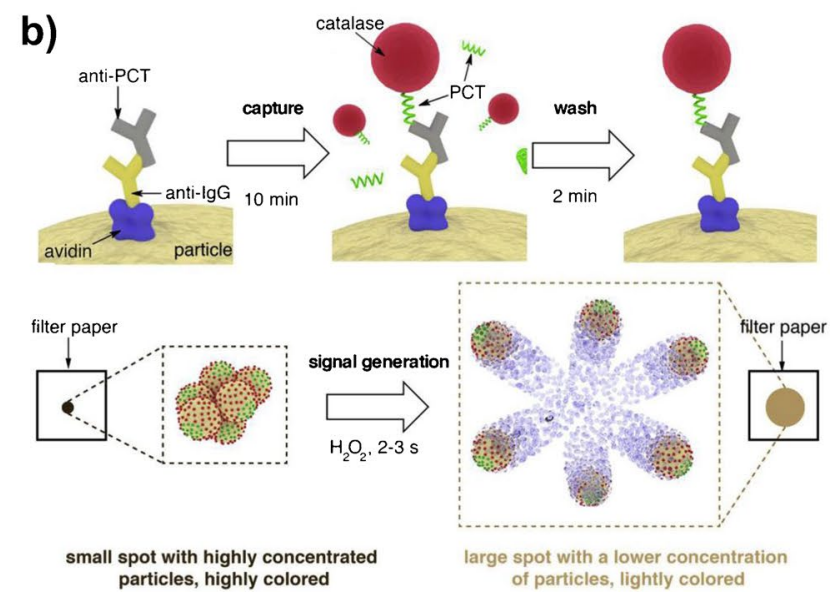

cific endotoxin (right). Bottom middle part are the corresponding fluorescence microcopy images of each step. b Anti-PCT modified iron oxide JMs modified with catalase for PCT detection. Reprinted with permission from ref. [58] (a) and ref (b) [60]

toxin achieving a LOD of $2 \mathrm{ng} / \mathrm{mL}$ and excellent selectivity in the presence of other toxins [61]. JMs using iron oxide have been modified with procalcitonin (PCT) antibodies for the detection of PCT in a paper-based assay. As can be seen in Fig. 9b, after the binding of PCT, the resulting complex was labelled with catalase for propulsion in the presence of peroxide. The JMs were dropped in a filter paper to facilitate color observation. In the presence of PCT, catalase was attached to the complex, thus generating oxygen bubbles for propulsion, and dispersing the color in the filter paper. Such changes were monitored with a smartphone, allowing the fast sensing of PCT for sepsis diagnosis [60].

Non-catalytic JMs have been very recently developed. Colloidal crystal-based stomatocyte micromotors can act as barcodes with different colors due to characteristic reflection
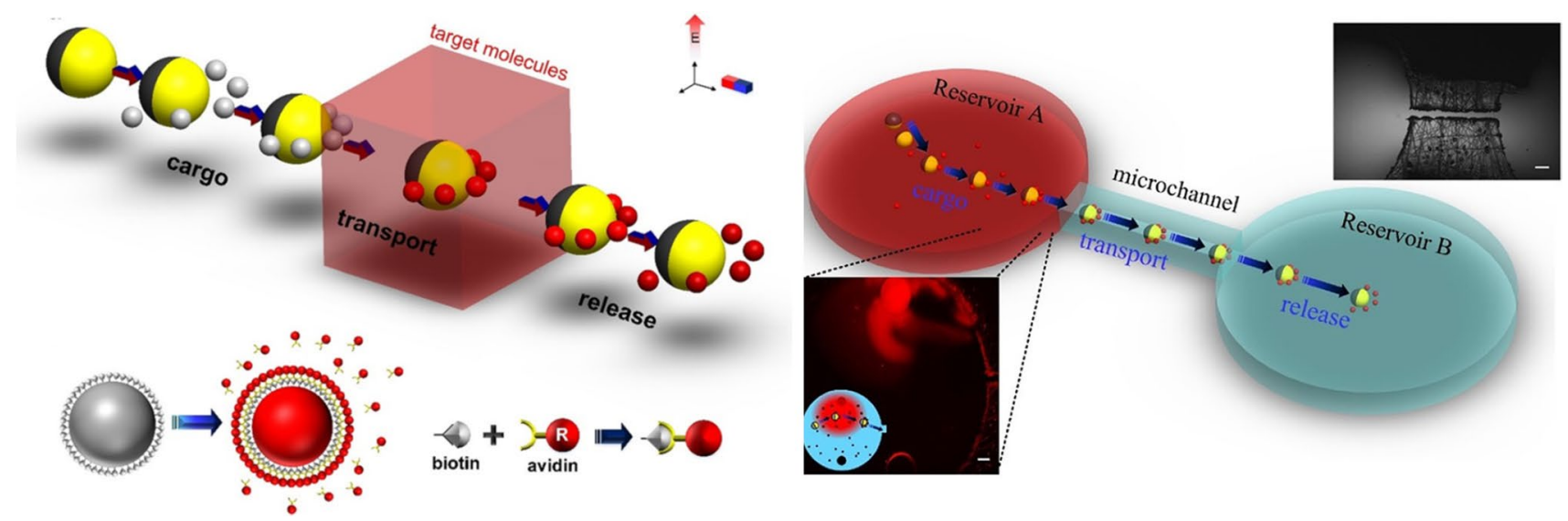

Fig. 10 Non-catalytic JMs for optical sensing. Concept schematic of magnetic JMs for label-free isolation and transport of biotin-avidin beads within the reservoirs of a microchip. Reprinted with permission from ref. [63] 
peaks for DNA detection [62]. A biosensing concept using magnetic micromotors is illustrated in Fig. 10. Polystyrene beads were half coated with $\mathrm{Cr} / \mathrm{Ni} / \mathrm{Au}$ layer for magnetic propulsion. The micromotors can be controlled by electric and magnetic fields for label-free capture of biotin-functionalized beads, which are transported within the reservoirs of a microchip for capture of avidin-functionalized cargo and subsequent release. Such concept allows performing bioassays even in whole blood with enhanced biocompatibility [63].

\section{Cell imaging and biosensing}

Due to their unique properties, JPs are attractive tools for optical imaging and sensing in biological systems [64] although their potential in this field has not been much explored.

Magnetically and optically active nanoparticles provide unique advantages for in vivo real-time cell imaging. In this context, magnetic QDs, which combine the optical properties of QDs to enable imaging of cells, with the magnetic ability of iron oxide nanoparticles to enable magnetic resonance imaging, are particularly interesting JPs. This type of particles has been explored by some authors allowing, with a single type of Janus nanoparticles, simultaneous magnetic resonance imaging (MRI) and optical reflection imaging $[65,66]$ and even cell-targeting capacity by functionalization with a specific antibody [67]. These are also examples of asymmetric structures where asymmetry is not really necessary.

Besides magnetic QD systems, plasmonic tadpole-like JPs with enhanced cellular internalization have been used as plasmonic probes for near infrared (NIR) cell imaging [68]. Matchstick-shaped $\mathrm{Au} / \mathrm{ZnO} / \mathrm{SiO}_{2}$ Janus nanorods, allowing multimodal dark-field, two-photon, and fluorescence imaging [69], Janus polymer nanospheres [70], and $\mathrm{Fe}-\mathrm{Au}$ nanorods [71] have also demonstrated potential in cell imaging.

Regarding cellular biosensing applications, the development of antibody-modified JPs for targeting and SERS sensing of tumor cells $[51,52]$ and JPs which combine sensing and barcoding functions to enable multiplexed bioanalysis [72-78] should be mentioned.

Regarding cell applications of JMs, there is no doubt that, due to their capacity for internalization without compromising cell viability, acoustically propelled nanomotors are well ahead of other motors for this kind of applications. During the last few years, US-propelled JMs have shown relevant intracellular applications [79, 80] for the sensing of biomarkers with high clinical interest (miRNA-21 [81], E6 mRNA transcripts [82], and AIB1 [83]) and delivery of therapeutics such as a small interfering RNA (siRNA) [84] and caspase-3
(CASP-3) [85] in a functional form for targeted silencing or apoptosis, respectively.

However, to our knowledge, Janus motors' forays into this field lag those of ultrasonic motors and have not yet demonstrated cellular application in biosensing or bioimaging.

\section{In vivo biosensing and bioimaging}

Despite the significant advances made in the construction of diverse nano and micromotors over the last decade and the booming research enthusiasm in this field [86], the requirement of cell-toxic fuels by many of such motors or the limited lifetime of motors with dissolving body (such as the magnesium ones) have hampered their use for in vivo applications [40]. However, recent developments in magnetic [87], ultrasound [88], and $\mathrm{Zn}$ and $\mathrm{Mg}$ micromotors [89] micromotors have shed some light on the field demonstrating promising proof-of-concept in vivo applications [90-93].

Indeed, different types of biocompatible and biodegradable chemically powered body-fuel-propelled micromotors have shown their potential for in vivo applications other than biosensing including precise micromotor tissue localization and retention, autonomous gastric fluid neutralization and cargo release, and improved drug delivery to enhance the treatment of bacterial infection of the stomach [89].

Although JMs have been evaluated extensively under in vitro conditions exhibiting interesting attributes including the possibility of fabrication with biocompatible materials (hollow MS NPs [94]), their motion consuming nontoxic fuels [94], in acidic environment produced by Hela cells [95] or blood [96] and self-destruction capabilities [30], JMs in vivo function has been little explored up to date.

Wang's group used different body fuel-propelled micromotors (Zn-based tubular micromotors, Mg-based JMs, and $\mathrm{Mg}$ microparticles-loaded tubular micromotors) for in vivo applications in the gastrointestinal tract profiting their autonomous driven by generating hydrogen bubbles through acid (Zn-based) or aqueous-acidic (Mg-based) solutions [89]. These body fuel-propelled micromotors have shown considerable promise for navigating through complex biological fluids, reaching hard-to-access body locations, and releasing their therapeutic payloads at predetermined destinations [90, 91, 93]. Indeed, JMs have been applied in vivo (mouse model) to safely and rapidly neutralize gastric acid through efficient chemical propulsion in the gastric fluid (Fig. 11b) by rapidly depleting the localized protons and simultaneously release payload without causing noticeable acute toxicity or affecting the stomach function [92], or to treat gastric bacterial (Helicobacter pylori) infection (Fig. 11c) [93] or iron deficiency anemia (Fig. 11d) [97] by delivery of drugs or minerals, respectively, more efficiently than using passive carriers. Although at an early stage, these results open the door to the use of synthetic motors as active-delivery 


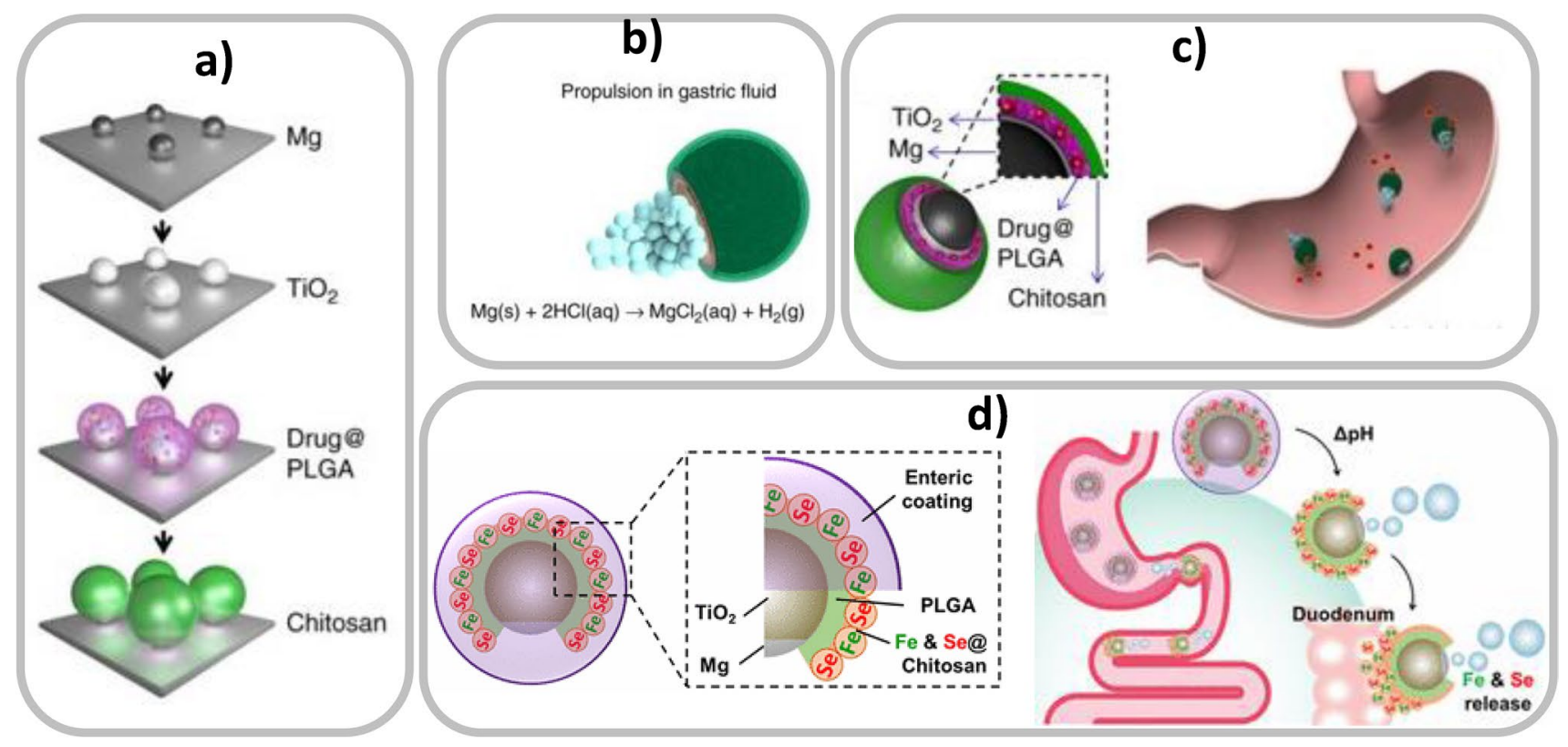

Fig. 11 a Synthesis of cargo-loaded Mg-based micromotors. b Propulsion of $\mathrm{Mg}$-based micromotors in acidic medium. c Schematic diagram of the $\mathrm{Mg} / \mathrm{TiO}_{2} / \mathrm{Drug} @ \mathrm{PLGA} /$ chitosan micromotor and propulsion and drug delivery in a mouse stomach and (d) schematic

platforms for in vivo treatment of diseases and leads one to believe that a more efficient in vivo biosensing will be the next step.

\section{Challenges and future perspectives}

JPs and JMs are materials that combine multifunctional properties in a single unit. This fact can greatly benefit biosensing by attaching capture probes in one part of the particles and other components, e.g., signal enhancers or substances to avoid biofouling and other interferences in complex samples, in the other part. An important aspect of JPs and JMs to be considered in biosensing is the choice of an adequate material and synthetic route to achieve high biocompatibility without compromising analytical performance. The large number of available materials and synthetic routes (self-assembly, masking, and phase separation) allows tailoring the JPs and JMs composition to obtain full biocompatibility in the analyses media or to get the desired analytical properties.

Several electrochemical biosensing approaches have been reported for the determination of clinical biomarkers using silica-Au and polymer-based micromotors as the most employed. However, catalytic JMs have been less used due to the interference of the fuel utilized to propel them with the electrochemical signal. Nevertheless, there is no doubt of the extraordinary potential of Mg-based micromotors structure of the $\mathrm{Mg} / \mathrm{TiO}_{2} / \mathrm{PLGA} / \mathrm{Fe}-\mathrm{Se} @$ chitosan/pH-responsive enteric coating micromotor and in vivo release of $\mathrm{Fe}$ and $\mathrm{Se}$ at the duodenum region. Reprinted and adapted from ref. (a-c) [93] and (d) [97]

for enhanced mixing and assisted degradation in strip electrodes. In this sense, more developments for electrochemical sensing can be expected by exploiting the properties of magnetic, light, or ultrasound-propelled micromotors.

Regarding optical detection, a lot of in vitro methods, mainly using fluorescence detection, involving both JPs and JMs, have been reported, for instance, for point-of-care diagnosis in sepsis or SARS-CoV-2 detection. Yet, in vivo methods and cell-based sensing still require more developments due to the difficulties in body biocompatibility and adequate imaging. Recent advances in this direction with degradable $\mathrm{Mg}$ micromotors or ultrasound-propelled nanorod hold considerable promise for in vivo detection of clinical biomarkers in the near future.

A general and expanded bird's eye view of the topics reviewed and discussed leads to the conclusion that there are many challenges to be addressed and space to be explored in this exciting field of a clear multidisciplinary nature.

Although JPs and JMs have emerged as new materials with exciting properties in the field of biosensing, many challenges currently hinder their widespread adoption in this and other disciplines. For example, further efforts are essential to develop simple fabrication techniques that enable the fabrication of JPs with defined functionality, morphology, and size distribution on a large scale and their biofunctionalization with high stability and adequate long-term storage in an environmentally friendly and low-cost manner. On the other hand, JPs with different surface chemistry offer more 
opportunities to combine unique features, but also introduce more complex interactions than uniform nanoparticles. A better understanding of these interactions is critical for the rational design and exploitation of JPs as new multifunctional tools. Their physical stability, biodistribution, and toxicity should also be studied more thoroughly.

As for JMs, the development of a direct method for onsite interpretation rather than measuring the velocity change calculated from various motors under microscope observation and the further exploitation of fuel-free and biocompatible JMs are included in the list of research worthwhile to address. This later would reduce the number of interferences primarily in electrochemical biosensing strategies and allow enhancing in vivo applications by fabricating JMs capable of evading immune system attack.

Moreover, the highlighted proof-of-concept applications demonstrate the applicability of multifunctional Janus structures for tailored analytical (bio)-sensing and the considerable promise hold by the asymmetric properties of JPs for a myriad of applications with the added advantage of easy surface modification without deactivation of catalytic activity and reusability use via magnetic separation. However, research in this field should devote efforts for improving the overall analytical performance to ensure compatibility with real-world applications and to perform comprehensive studies showing the advantages of Janus nanomaterials compared to the methods that use symmetrical or related nanostructures.

It is obvious that the great leap of these devices from the laboratory to the market must most likely pass through standardizing and improving the fabrication and modification of the JPs and JMs making detection results more repeatable and their motion more stable; exploring new power sources to improve their prolonged navigation and penetration for fully autonomous operation in complex environments; working on the control and monitoring of wireless micromotors through high-resolution imaging and their coupling with equipment normally used for biomedical diagnostics or cell phones with integrated signal reading; performing more comprehensive biocompatibility studies to ensure compatibility with in vivo applications; and certainly dare to explore their potential far beyond proof-of-concept applications.

Despite these significant barriers to overcome, due to their unique characteristics and reported pioneering applications, JPs and JMs are favored to tackle analytical strategies that have long been considered unattainable and now limited only by our imagination. Thus, it can be predicted, with all sense that in the coming years, we will witness JPs- and JMs-assisted research demonstrating increased prominence of enzyme-functionalized nanomotors driven by constituents of body fluids; new developments further exploiting bioaffinity reactions for new portable electroanalytical systems integrated into cell phones, or even wearable sensors for rapid disease diagnosis, environmental analysis, or food quality assurance; and empowering their applications for sensing and biosensing on microfluidic chips, microliter solutions, and microenvironments poorly compatible with more conventional sensing and biosensing technologies.

Acronyms CASP-3: Caspase-3; CBCPHNs: Compartmentalized bimetal cluster-poly(aniline) hybrid nanostructures; CEA: Carcinoembryonic antigen; CNTs: Carbon nanotubes; CV: Cyclic voltammetry; DA: Dopamine; DCP: Diethyl chlorophosphite; DPP: Diphenyl phthalate; DPV: Differential pulse voltammetry; EDC/NHS: Carbodiimide/ succinimide chemistry; FLA: Fluorescein amine; GCE: Glassy-carbon electrode; GOx: Glucose oxidase; HQ: Hydroquinone; HRP: Horseradish peroxidase; JMs: Janus micromotors; JPs: Janus particles; KDO: 3-Deoxy-d-manno-octulosonic acid; LOD: Limit of detection; MGITC: Malachite green isothiocyanate; MRI: Magnetic resonance imaging; MS: Mesoporous silica; NIR: Near-infrared; NPs: Nanoparticles; OP: Organophosphorous; OTA: Ochratoxin A; PA: Polyaniline; PCL: Polycaprolactone; PCT: Procalcitonin; QDs: Quantum dots; RAC: Ractopamine; RBITC: Rhodamine B isothiocyanate; SERS: Surface-enhanced Raman scattering; SiRNA: Small interfering RNA; SPCE: Screen-printed carbon electrode; STv: Streptavidin; TEM: Transmission-electron microscopy; TMB: 3,3',5,5'-Tetramethylbenzidine; $\mathrm{TOA}^{+}$: Tetraoctylammonium cation

Author contribution Conceptualization, S.C, J.M.P., B.J.S., and A.E.; writing-original draft preparation, S.C., B.J.S.; writing-review and editing, S.C, J.M.P., B.J.S., and A.E. Funding acquisition, S.C, J.M.P., B.J.S., and A.E.

Funding This research was funded by the Spanish Ministry of Science and Innovation, grant number RYC-2015-17558 co-financed by EU (B.J.S); the Spanish Ministry of Science and Innovation, grant numbers PID2020-118154 GB-I00 (A. E and B.J.S; Funded by MICIN/ AEI/10.13039/501100011033) and PID2019-103899RB-I00 (S.C.); and the Community of Madrid, grant number TRANSNANOAVANSENS, S2018/NMT-4349 (A.E, S.C), and CM/JIN/2019-007 (B.J.S).

\section{Declarations}

Conflict of interest The authors declare no competing interests.

\section{References}

1. Zhang X, Fu Q, Duan H, Song J, Yang H (2021) Janus nanoparticles: from fabrication to (bio)applications. ACS Nano 15:6147-6191

2. de Gennes PG (1992) Soft Matter. Science 256:495-497

3. Pourrahimi AM, Pumera M (2018) Multifunctional and selfpropelled spherical Janus nano/micromotors: recent advances. Nanoscale 10:16398-16415

4. Ye Y, Luan J, Wang M, Chen Y, Wilson DA, Peng F et al (2019) Fabrication of self-propelled micro- and nanomotors based on Janus structures. Chem Eur J 25:8663-8680

5. Jurado-Sánchez B, Pacheco M, Maria-Hormigos R, Escarpa A (2017) Perspectives on Janus micromotors: materials and applications. Appl Mater Tod 9:407-418 
6. Yánez-Sedeño P, Campuzano S, Pingarrón JM (2017) Janus particles for (bio)sensing. Appl Mater Tod 9:276-288

7. Rahiminezhad Z, Tamaddon AM, Borandeh S, Abolmaali SS (2020) Janus nanoparticles: new generation of multifunctional nanocarriers in drug delivery, bioimaging and theranostics. Appl Mater Tod 18:100513

8. Biji P, Patnaik A (2012) Interfacial Janus gold nanoclusters as excellent phase- and orientation-specific dopamine sensors. Analyst 137:4795-4801

9. Singh VV, Kaufmann K, Orozco J, Li J, Galarnyk M, Arya G et al (2015) Micromotor-based on-off fluorescence detection of sarin and soman simulants. Chem Commun 51:11190-11193

10. Nie L, Liu S, Shen W, Chen D, Jiang M (2007) One-Pot synthesis of amphiphilic polymeric janus particles and their selfassembly into supermicelles with a narrow size distribution. Angew Chem Int Ed 46(33):6321-6324

11. Yuan K, de la Asunción-Nadal V, Jurado-Sánchez B, Escarpa A (2020) 2D Nanomaterials wrapped janus micromotors with built-in multiengines for bubble, magnetic, and light driven propulsion. Chem Mater 32:1983-1992

12 Safaie N, Ferrier RC Jr (2020) Janus nanoparticle synthesis: overview, recent developments, and applications. J Appl Phys 127:170902

13. Du J, O'Reilly RK (2011) Anisotropic particles with patchy, multicompartment and Janus architectures: preparation and application. Chem Soc Rev 40:2402-2416

14. Pawar AB, Kretzschmar I (2009) Multifunctional patchy particles by glancing angle deposition. Langmuir 25:9057-9063

15. Cinti S, Valdés-Ramírez G, Gao W, Li J, Palleschi G, Wang J (2015) Microengine-assisted electrochemical measurements at printable sensor strips. Chem Commun 51(41):8668-8671

16. Böker A, He J, Emrick T, Russell TP (2007) Self-assembly of nanoparticles at interfaces. Soft Matter 3:1231-1248

17. Kaewsaneha C, Tangboriboonrat P, Polpanich D, Eissa M, Elaissari A (2013) Preparation of Janus colloidal particles via Pickering emulsion: an overview. Coll Surf A 439:35-42

18. Hong L, Jiang S, Granick S (2006) Simple method to produce janus colloidal particles in large quantity. Langmuir 22:9495-9499

19. Martens S, Holloway JO, Du Prez FE (2017) Click and clickinspired chemistry for the design of sequence-controlled polymers. Macromol Rap Commun 38:1700469

20. Lone S, Kim SH, Nam SW, Park S, Joo J, Cheong IW (2011) Microfluidic synthesis of Janus particles by UV-directed phase separation. Chem Commun 47:2634-2636

21. Liu B, Möhwald H, Wang D (2013) Synthesis of Janus particles via kinetic control of phase separation in emulsion droplets. Chem Commun 49:9746-9748

22. Tanaka T, Nakatsuru R, Kagari Y, Saito N, Okubo M (2008) Effect of molecular weight on the morphology of polystyrene/ poly(methyl methacrylate) composite particles prepared by the solvent evaporation method. Langmuir 24:12267-12271

23. Jurado-Sánchez B, Pacheco M, Rojo J, Escarpa A (2017) Magnetocatalytic graphene quantum dots janus micromotors for bacterial endotoxin detection. Angew Chem Int Ed 56:6957-6961

24. Loget G, Roche J (2012) Kuhn A (2012) True bulk synthesis of Janus objects by bipolar electrochemistry. Adv Mater 24:5111-5116

25. Marschelke C, Fery A, Synytska A (2020) Janus particles: from concepts to environmentally friendly materials and sustainable applications. Colloid Polym Sci 298:841-865

26. Zhao LB, Pan L, Zhang K, Guo SS, Liu W, Wang Y et al (2009) Generation of Janus alginate hydrogel particles with magnetic anisotropy for cell encapsulation. Lab Chip 9:2981-2986

27. Hu Y, Wang S, Abbaspourrad A, Ardekani AM (2015) Fabrication of shape controllable janus alginate/pnipaam microgels via microfluidics technique and off-chip ionic cross-linking. Langmuir 31:1885-1891

28. Sun X-T, Zhang Y, Zheng D-H, Yue S, Yang C-G, Xu Z-R (2017) Multitarget sensing of glucose and cholesterol based on Janus hydrogel microparticles. Biosens Bioelectron 92:81-86

29. Gao W, Liu M, Liu L, Zhang H, Dong B, Li CY (2015) Onestep fabrication of multifunctional micromotors. Nanoscale 7:13918-13923

30. Chen C, Karshalev E, Li J, Soto F, Castillo R, Campos I et al (2016) Transient micromotors that disappear when no longer needed. ACS Nano 10:10389-10396

31. Chen C, Karshalev E, Guan J, Wang J (2018) Magnesium-based micromotors: water-powered propulsion, multifunctionality, and biomedical and environmental applications. Small 14:1704252

32. Pacheco M, Jurado-Sánchez B, Escarpa A (2019) Visible-lightdriven janus microvehicles in biological media. Angew Chem Int Ed 58:18017-18024

33. Landgraf L, Christner C, Storck W, Schick I, Krumbein I, Dähring $\mathrm{H}$ et al (2015) A plasma protein corona enhances the biocompatibility of $\mathrm{Au} @ \mathrm{Fe}_{3} \mathrm{O}_{4}$ Janus particles. Biomaterials 68:77-88

34. Tang JL, Schoenwald K, Potter D, White D, Sulchek T (2012) Bifunctional janus microparticles with spatially segregated proteins. Langmuir 28(26):10033-10039

35. Wu J, Zhang D, He X, Wang Y, Xiao S, Chen F et al (2019) "Janus-Featured" hydrogel with antifouling and bacteria-releasing properties. Ind Eng Chem Res 58:17792-17801

36. Jurado-Sánchez B, Escarpa A (2017) Janus micromotors for electrochemical sensing and biosensing applications: a review. Electroanalysis 29:14-23

37. Kong L, Guan J, Pumera M (2018) Micro- and nanorobots based sensing and biosensing. Curr Opin Electrochem 10:174-182

38. Campuzano S, Gamella M, Serafín V, Pedrero M, Yáñez-Sedeño P, Pingarrón JM (2019) Magnetic janus particles for static and dynamic (bio)sensing. Magnetochemistry 5:47

39. Zhou Y, Yang Y, Deng X, Zhang G, Zhang Y, Zhang $C$ et al (2018) Electrochemical sensor for determination of ractopamine based on aptamer/octadecanethiol Janus particles. Sens Actuat B 276:204-210

40. Pacheco M, López MÁ, Jurado-Sánchez B, Escarpa A (2019) Self-propelled micromachines for analytical sensing: a critical review. Anal Bioanal Chem 411:6561-6573

41. Boujakhrout A, Sánchez E, Díez P, Sánchez A, Martínez-Ruiz P, Parrado C et al (2015) Single-walled carbon nanotubes/aumesoporous silica janus nanoparticles as building blocks for the preparation of a bienzyme biosensor. ChemElectroChem 2:1735-1741

42. Sánchez A, Díez P, Martínez-Ruíz P, Villalonga R, Pingarrón JM (2013) Janus Au-mesoporous silica nanoparticles as electrochemical biorecognition-signaling system. Electrochem Communi 30:51-54

43. Paniagua G, Villalonga A, Eguílaz M, Vegas B, Parrado C, Rivas $\mathrm{G}$ et al (2019) Amperometric aptasensor for carcinoembryonic antigen based on the use of bifunctionalized Janus nanoparticles as biorecognition-signaling element. Anal Chim Acta 1061:84-91

44. Yang Y-J, Zhou Y, Xing Y, Zhang G-M, Zhang Y, Zhang C-H et al (2019) A Label-free aptasensor based on Aptamer/ $\mathrm{NH}_{2}$ Janus particles for ultrasensitive electrochemical detection of Ochratoxin A. Talanta 199:310-316

45. Morales-Narváez E, Guix M, Medina-Sánchez M, Mayorga-Martinez CC, Merkoçi A (2014) Micromotor enhanced microarray technology for protein detection. Small 10:2542-2548

46. Rojas D, Jurado-Sánchez B, Escarpa A (2016) "Shoot and Sense" janus micromotors-based strategy for the simultaneous degradation and detection of persistent organic pollutants in food and biological samples. Anal Chem 88:4153-4160 
47. Kong L, Rohaizad N, Nasir MZM, Guan J, Pumera M (2019) Micromotor-assisted human serum glucose biosensing. Anal Chem 91:5660-5666

48. Salinas G, Dauphin AL, Voci S, Bouffier L, Sojic N, Kuhn A (2020) Asymmetry controlled dynamic behavior of autonomous chemiluminescent Janus microswimmers. Chem Sci 11:7438-7443

49. Moo JGS, Pumera M (2016) Self-propelled micromotors monitored by particle-electrode impact voltammetry. ACS Sens 1(7):949-957

50. Khezri B, Sheng Moo JG, Song P, Fisher AC, Pumera M (2016) Detecting the complex motion of self-propelled micromotors in microchannels by electrochemistry. RSC Adv 6:99977-99982

51. Wu LY, Ross BM, Hong S, Lee LP (2010) Bioinspired nanocorals with decoupled cellular targeting and sensing functionality. Small 6:503-507

52. Hsieh H-Y, Huang T-W, Xiao J-L, Yang C-S, Chang C-C, Chu C-C et al (2012) Fabrication and modification of dual-faced nano-mushrooms for tri-functional cell theranostics: SERS/ fluorescence signaling, protein targeting, and drug delivery. J Mater Chem 22:20918-20928

53. Hwang EY, Lee JH, Lim DW (2020) Compartmentalized bimetal cluster-poly(aniline) hybrid nanostructures for multiplexed detection of autoantibodies in early diagnosis of rheumatoid arthritis. Sens Actuat B 321:128482

54. Lu C, Liu X, Li Y, Yu F, Tang L, Hu Y et al (2015) Multifunctional janus hematite-silica nanoparticles: mimicking peroxidase-like activity and sensitive colorimetric detection of glucose. ACS Appl Mater Interfac 7:15395-15402

55. Li J, Savagatrup S, Nelson Z, Yoshinaga K, Swager TM (2020) Fluorescent Janus emulsions for biosensing of Listeria monocytogenes. Proc Nat Ac Sci 117:11923-11930

56. Li J, Concellón A, Yoshinaga K, Nelson Z, He Q, Swager TM (2021) Janus emulsion biosensors for anti-SARS-CoV-2 spike antibody. ACS Central Sci 7:1166-1175

57. Pacheco M, Jurado-Sánchez B, Escarpa A (2018) Sensitive monitoring of enterobacterial contamination of food using selfpropelled janus microsensors. Anal Chem 90(4):2912-2917

58. Pacheco M, Asunción-Nadal V, Jurado-Sánchez B, Escarpa A (2020) Engineering Janus micromotors with $\mathrm{WS}_{2}$ and affinity peptides for turn-on fluorescent sensing of bacterial lipopolysaccharides. Biosens Bioelectron 165:112286

59. Wang K, Wang W, Pan S, Fu Y, Dong B, Wang H (2020) Fluorescent self-propelled covalent organic framework as a microsensor for nitro explosive detection. Appl Mater Tod 19:100550

60. Russell SM, Alba-Patiño A, Borges M, de la Rica R (2019) Multifunctional motion-to-color janus transducers for the rapid detection of sepsis biomarkers in whole blood. Biosen Bioelectron 140:111346

61. Yuan K, López MÁ, Jurado-Sánchez B, Escarpa A (2020) Janus micromotors coated with $2 \mathrm{D}$ nanomaterials as dynamic interfaces for (bio)-sensing. ACS Appl Mater Interfac 12:46588-46597

62. Cai L, Wang H, Yu Y, Bian F, Wang Y, Shi K et al (2019) Stomatocyte structural color-barcode micromotors for multiplex assays. Nat Sci Rev 7:644-651

63. Park S, Yossifon G (2020) Micromotor-based biosensing using directed transport of functionalized beads. ACS Sens 5:936-942

64. Yi Y, Sanchez L, Gao Y, Yu Y (2016) Janus particles for biological imaging and sensing. Analyst 141:3526-3539

65. Selvan ST, Patra PK, Ang CY, Ying JY (2007) Synthesis of silicacoated semiconductor and magnetic quantum dots and their use in the imaging of live cells. Angew Chem Int Ed 46:2448-2452

66. Lee J, Hwang G, Hong YS, Sim T (2015) One step synthesis of quantum dot-magnetic nanoparticle heterodimers for dual modal imaging applications. Analyst 140:2864-2868
67. Xu C, Xie J, Ho D, Wang C, Kohler N, Walsh EG et al (2008) Au$\mathrm{Fe}_{3} \mathrm{O}_{4}$ Dumbbell nanoparticles as dual-functional probes. Angew Chem Int Ed 47:173-176

68. Kim S, Yoo J-B, Yi G-R, Lee Y, Choi HR, Koo JC et al (2014) An aggregation-mediated assembly of graphene oxide on aminefunctionalized poly(glycidyl methacrylate) microspheres for coreshell structures with controlled electrical conductivity. J Mater Chem C 2:6462-6466

69. Susewind M, Schilmann A-M, Heim J, Henkel A, Link T, Fischer K et al (2015) Silica-coated Au@ZnO Janus particles and their stability in epithelial cells. J Mater Chem B 3:1813-1822

70. Hu S-H, Gao X (2010) Nanocomposites with spatially separated functionalities for combined imaging and magnetolytic therapy. $\mathrm{J}$ Am Chem Soc 132:7234-7237

71. Kilinc D, Lesniak A, Rashdan SA, Gandhi D, Blasiak A, Fannin PC et al (2015) Mechanochemical stimulation of MCF-7 cells with rod-shaped $\mathrm{Fe}-\mathrm{Au}$ janus particles induces cell death through paradoxical hyperactivation of ERK. Adv Healthcare Mater 4:395-404

72. Nicewarner-Peña SR, Freeman RG, Reiss BD, He L, Peña DJ, Walton ID et al (2001) Submicrometer metallic barcodes. Science 294:137-141

73. Nicewarner-Peña SR, Carado AJ, Shale KE, Keating CD (2003) Barcoded metal nanowires: optical reflectivity and patterned fluorescence. J Phys Chem B 107:7360-7367

74. Pregibon DC, Toner M, Doyle PS (2007) Multifunctional encoded particles for high-throughput biomolecule analysis. Science 315(5817):1393-1396

75. Bong KW, Chapin SC, Doyle PS (2010) Magnetic barcoded hydrogel microparticles for multiplexed detection. Langmuir 26(11):8008-8014. https://doi.org/10.1021/la904903g

76. Yuet KP, Hwang DK, Haghgooie R, Doyle PS (2010) Multifunctional superparamagnetic Janus particles. Langmuir 26:4281-4287

77. Appleyard DC, Chapin SC, Srinivas RL, Doyle PS (2011) Barcoded hydrogel microparticles for protein detection: synthesis, assay and scanning. Nat Protoc 6:1761-1774

78. Lee J, Bisso PW, Srinivas RL, Kim JJ, Swiston AJ, Doyle PS (2014) Universal process-inert encoding architecture for polymer microparticles. Nat Mater 13:524-529

79 Campuzano S, Esteban-Fernández de Ávila B, Yáñez-Sedeño P, Pingarrón JM, Wang J (2017) Nano/microvehicles for efficient delivery and (bio)sensing at the cellular level. Chem Sci 8:6750-6763

80 Venugopalan PL, Esteban-Fernández de Ávila B, Pal M, Ghosh A, Wang J (2020) Fantastic voyage of nanomotors into the cell. ACS Nano 14:9423-39

81. Esteban-Fernández de Ávila B, Martín A, Soto F, Lopez-Ramirez MA, Campuzano S, Vásquez-Machado GM et al (2015) Single cell real-time miRNAs sensing based on nanomotors. ACS Nano 9:6756-6764

82. Qualliotine JR, Bolat G, Beltrán-Gastélum M, de Ávila BE-F, Wang J, Califano JA (2019) Acoustic nanomotors for detection of human papillomavirus-associated head and neck cancer. Otolaryngol Head Neck Surg 161:814-822

83. Beltrán-Gastélum M, Esteban-Fernández de Ávila B, Gong H, Venugopalan PL, Hianik T, Wang J et al (2019) Rapid Detection of AIB1 in breast cancer cells based on aptamer-functionalized nanomotors. ChemPhysChem 20:3177-3180

84. Esteban-Fernández de Ávila B, Angell C, Soto F, Lopez-Ramirez MA, Báez DF, Xie S et al (2016) Acoustically propelled nanomotors for intracellular siRNA delivery. ACS Nano 10:4997-5005

85 Esteban-Fernández de Ávila B, Ramírez-Herrera DE, Campuzano S, Angsantikul P, Zhang L, Wang J (2017) Nanomotor-enabled pH-responsive intracellular delivery of caspase-3: toward rapid cell apoptosis. ACS Nano 11:5367-5374 
86. Peng F, Tu Y, Wilson DA (2017) Micro/nanomotors towards in vivo application: cell, tissue and biofluid. Chem Soc Rev 46(17):5289-5310

87. Chen X-Z, Hoop M, Mushtaq F, Siringil E, Hu C, Nelson BJ et al (2017) Recent developments in magnetically driven micro- and nanorobots. Appl Mater Tod 9:37-48

88. Xu T, Xu L-P, Zhang X (2017) Ultrasound propulsion of micro-/ nanomotors. Appl Mater Tod 9:493-503

89 Esteban-Fernández de Ávila B, Angsantikul P, Li J, Gao W, Zhang L, Wang J (2018) Micromotors go in vivo: from test tubes to live animals. Adv Function Mater 28:1705640

90. Gao W, Dong R, Thamphiwatana S, Li J, Gao W, Zhang L et al (2015) Artificial micromotors in the mouse's stomach: a step toward in vivo use of synthetic motors. ACS Nano 9:117-123

91. Li J, Thamphiwatana S, Liu W, Esteban-Fernández de Ávila B, Angsantikul P, Sandraz E et al (2016) Enteric micromotor can selectively position and spontaneously propel in the gastrointestinal tract. ACS Nano 10:9536-9542

92. Li J, Angsantikul P, Liu W, Esteban-Fernández de Ávila B, Thamphiwatana S, Xu M et al (2017) Micromotors spontaneously neutralize gastric acid for ph-responsive payload release. Angew Chem Int Ed 56:2156-61

93. Esteban-Fernández de Ávila B, Angsantikul P, Li J, Angel LopezRamirez M, Ramírez-Herrera DE, Thamphiwatana S et al (2017)
Micromotor-enabled active drug delivery for in vivo treatment of stomach infection. Nat Commun 8:272

94. Ma X, Jannasch A, Albrecht U-R, Hahn K, Miguel-López A, Schäffer E et al (2015) Enzyme-powered hollow mesoporous janus nanomotors. Nano Lett 15:7043-7050

95. Guix M, Meyer AK, Koch B, Schmidt OG (2016) Carbonatebased Janus micromotors moving in ultra-light acidic environment generated by HeLa cells in situ. Sci Rep 6:21701

96. Mou F, Chen C, Zhong Q, Yin Y, Ma H, Guan J (2014) Autonomous motion and temperature-controlled drug delivery of $\mathrm{Mg}$ / Pt-Poly(N-isopropylacrylamide) Janus micromotors driven by simulated body fluid and blood plasma. ACS Appl Mater Interfac 6:9897-9903

97. Karshalev E, Zhang Y, Esteban-Fernández de Ávila B, BeltránGastélum M, Chen Y, Mundaca-Uribe R et al (2019) Micromotors for active delivery of minerals toward the treatment of iron deficiency anemia. Nano Lett 19(11):7816-7826

Publisher's note Springer Nature remains neutral with regard to jurisdictional claims in published maps and institutional affiliations. 